PROCEEDINGS OF THE

AMERICAN MATHEMATICAL SOCIETY

Volume 131, Number 1, Pages 147-154

S 0002-9939(02)06587-5

Article electronically published on May 22, 2002

\title{
JORDAN ISOMORPHISMS OF NEST ALGEBRAS
}

\author{
FANGYAN LU
}

(Communicated by David R. Larson)

\begin{abstract}
Let $\mathcal{T}(N)$ and $\mathcal{T}(\mathcal{M})$ be two nest algebras. A Jordan isomorphism $\phi$ from $\mathcal{T}(N)$ onto $\mathcal{T}(\mathcal{M})$ is a bijective linear map such that $\phi\left(T^{2}\right)=\phi(T)^{2}$ for every $T \in \mathcal{T}(\mathcal{N})$. In this note, we prove that every Jordan isomorphism of nest algebras is of the form $T \rightarrow S T S^{-1}$ or $T \rightarrow S T^{*} S^{-1}$ and then is, in fact, an isomorphism or an anti-isomorphism.
\end{abstract}

The motivation for this paper is the work by J. Arazy and B. Solel. In 1, J. Arazy and B. Solel proved that every surjective isometry $\alpha$ of nest algebras is of the form $T \rightarrow U T U^{-1}$ or $T \rightarrow U T^{*} U^{-1}$ provided that $\alpha(I)=I$, where $U$ is a unitary operator. This is an elegant characterization. As we observed, they in fact first proved that such an isometry is a Jordan isomorphism and then completed their job. Let $\mathcal{T}(N)$ and $\mathcal{T}(\mathcal{M})$ be two nest algebras. A Jordan isomorphism $\phi$ from $\mathcal{T}(N)$ onto $\mathcal{T}(\mathcal{M})$ is a bijective linear map such that $\phi\left(T^{2}\right)=\phi(T)^{2}$ for every $T \in$ $\mathcal{T}(\mathcal{N})$. The aim of the present paper is to characterize Jordan isomorphisms of nest algebras. Our main result is that every Jordan isomorphism of nest algebras is of the form $T \rightarrow S T S^{-1}$ or $T \rightarrow S T^{*} S^{-1}$ and then is, in fact, either an isomorphism or an anti-isomorphism. The same result was concluded in [6] for Jordan isomorphisms from a ring onto an integral domain. Clearly a nest algebra is not an integral domain and a Jordan isompohism is not isometric; we must use different techniques. This leads us to study nilpotent Jordan ideals, which is the main subject of this paper.

Throughout, $\mathcal{H}$ is a complex Hilbert space, $B(\mathcal{H})$ is the algebra of all linear bounded operators on $\mathcal{H}, \mathcal{N}$ and $\mathcal{M}$ are nests of projections on $\mathcal{H}, \mathcal{T}(N)$ and $\mathcal{T}(\mathcal{M})$ are the nest algebras associated with $\mathcal{N}$ and $\mathcal{M}$ respectively, and $\phi$ is a Jordan isomorphism from $\mathcal{T}(\mathcal{N})$ onto $\mathcal{T}(\mathcal{M})$. For $N \in \mathcal{N}$, we use $N^{\perp}$ to denote $I-N$. For more information concerning nest algebras, we refer readers to [3].

We begin with two lemmas. The first is due to [6] and the second is well-known.

Lemma 1. For any $A, B, C \in \mathcal{T}(\mathcal{N})$, the following hold:

(1) $\phi(A B+B A)=\phi(A) \phi(B)+\phi(B) \phi(A)$.

(2) $\phi(A B A)=\phi(A) \phi(B) \phi(A)$.

(3) $\phi(A B C+C B A)=\phi(A) \phi(B) \phi(C)+\phi(C) \phi(B) \phi(A)$.

Lemma 2. We have $\mathcal{T}(\mathcal{N})^{\prime}=\mathbb{C} I$, where $\mathcal{T}(\mathcal{N})^{\prime}$ is the commutant of $\mathcal{T}(\mathcal{N})$ and $\mathbb{C}$ is the set of complex numbers.

Received by the editors June 8, 2000 and, in revised form, October 30, 2000 and August 16, 2001

2000 Mathematics Subject Classification. Primary 47L35, 47L20, 46H10.

Key words and phrases. Jordan isomorphism, nest algebra, nilpotent Jordan ideal.

(C)2002 American Mathematical Society 
Proposition 3. We have $\phi(I)=I$.

Proof. By Lemma 1, for every $T \in \mathcal{T}(\mathcal{N})$, we have that

$$
2 \phi(T)=\phi(T I+I T)=\phi(T) \phi(I)+\phi(I) \phi(T)
$$

and

$$
\phi(T)=\phi(I T I)=\phi(I) \phi(T) \phi(I) .
$$

Since $\phi(I)$ is an idempotent, by (1) and (2), we have that $\phi(T) \phi(I)=\phi(T)=$ $\phi(I) \phi(T)$. Therefore $\phi(I) \in \mathcal{T}(\mathcal{M})^{\prime}$, and then there exists a scalar $\lambda$ such that $\phi(I)=\lambda I$. Thus the result follows from the fact that $\phi(I)$ is an idempotent and $\phi(I) \neq 0$.

Proposition 4. Suppose that $T$ and $S$ are in $\mathcal{T}(\mathcal{N})$ such that $T S=S T=0$. Then $\phi(T) \phi(S)=\phi(S) \phi(T)=0$.

Proof. By Lemma 1(1), we have that

$$
\phi(T) \phi(S)+\phi(S) \phi(T)=\phi(T S+S T)=0 .
$$

For every $A \in \mathcal{T}(\mathcal{N})$, by Lemma $1(3)$,

$$
\phi(T) \phi(S) \phi(A)+\phi(A) \phi(S) \phi(T)=\phi(T S A+A S T)=0 .
$$

Combining (3) and (4) yields

$$
\phi(T) \phi(S) \phi(A)-\phi(A) \phi(T) \phi(S)=0 .
$$

Since $A$ is arbitrary, $\phi(T) \phi(S) \in \mathcal{T}(\mathcal{M})^{\prime}$. Hence

$$
\phi(T) \phi(S)=\lambda I
$$

for some scalar $\lambda$. Thus

$$
0=\phi(T S T)=\phi(T) \phi(S) \phi(T)=\lambda \phi(T) .
$$

Equalities (5) and (6) force $\phi(T) \phi(S)=0$ and then $\phi(S) \phi(T)=0$.

Let $\mathcal{S}$ be a subset of a Banach algebra $\mathcal{A}$. If $A B=B A=0$ for any $A, B \in \mathcal{S}$, we say that $\mathcal{S}$ is nilpotent. Proposition 4 shows that a Jordan isomorphism preserves nilpotent sets. Let $\mathcal{N}_{0}=\{N \in \mathcal{N}: 0<N<I\}$ and $\mathcal{M}_{0}=\{M \in \mathcal{M}: 0<M<I\}$. For $N \in \mathcal{N}_{0}$, let $\mathcal{I}(N)=\left\{N T N^{\perp}: T \in \mathcal{T}(\mathcal{N})\right\}$. Then $\mathcal{I}(N)$ is a nilpotent subset of $\mathcal{T}(\mathcal{N})$. Moreover, we will show that such $\mathcal{I}(N)$ is maximal in the sense that $\mathcal{I}(N)$ is not properly contained in any other nilpotent subset of $\mathcal{T}(\mathcal{N})$.

Lemma 5. If $\mathcal{S}$ is a nilpotent subset of $\mathcal{T}(\mathcal{N})$ such that $\mathcal{S} \supset \mathcal{I}(N)$ for some $N \in$ $\mathcal{N}_{0}$, then $\mathcal{S}=\mathcal{I}(N)$.

Proof. Suppose that $S \in \mathcal{S}$. Then for every $T \in \mathcal{T}(\mathcal{N})$, we have

$$
S N T N^{\perp}=0 \text { and } N T N^{\perp} S=0 .
$$

Since $S \in \mathcal{T}(\mathcal{N})$,

$$
N S N T N^{\perp}=0 \text { and } N T N^{\perp} S N^{\perp}=0 .
$$

Since $T$ is arbitrary, $N S N=N^{\perp} S N^{\perp}=0$. Hence $S=N S N^{\perp} \in \mathcal{I}(N)$.

$\mathcal{I}(N)$ is also an ideal, but in general, a Jordan isomorphism does not preserve ideals. For our purpose, we need the following weaker concept [6]. 
Definition 6. Let $\mathcal{J}$ be a subspace of $\mathcal{T}(\mathcal{N}) . \mathcal{J}$ is called a J-ideal (Jordan ideal) if $A T+T A \in \mathcal{J}$ for every $A \in \mathcal{J}$ and $T \in \mathcal{T}(\mathcal{N})$.

By Lemma 1, Jordan isomorphisms preserve J-ideals. By Lemma $5, \mathcal{I}(N)$ is a maximal nilpotent J-ideal. We will show that the ideals $\mathcal{I}(N)$ are a model for maximal nilpotent J-ideals. In what follows, the rank one operator $e \otimes f$ is defined by $(e \otimes f) x=(x, f) e$. For $N \in \mathcal{N}$, define $N_{-}=\sup \{P \in \mathcal{N}: P<N\}$. It is well known that $e \otimes f$ belongs to $\mathcal{T}(\mathcal{N})$ if and only if there is an element $N$ in $\mathcal{N}$ such that $e \in N \mathcal{H}$ and $f \in N_{-}^{\perp} \mathcal{H}$.

Theorem 7. Suppose that $\mathcal{J}$ is a maximal nilpotent ideal of $\mathcal{T}(\mathcal{N})$. Then there exists an element $N$ in $\mathcal{N}_{0}$ such that $\mathcal{J}=\mathcal{I}(N)$.

Proof. Define

$$
\begin{aligned}
& N=\inf \left\{L \in \mathcal{N}: L^{\perp} \mathcal{J}=\{0\}\right\}, \\
& M=\sup \{L \in \mathcal{N}: \mathcal{J} L=\{0\}\} .
\end{aligned}
$$

We first prove that $N \leq M$. Otherwise $N>M$. Then we can take $T, S \in \mathcal{J}$ and vectors $e, f$ such that $e \otimes f \in \mathcal{T}(\mathcal{N})$ and $T e \otimes f S \neq 0$ as follows. If $M=N_{-}$, by the definition of $N$ and $M$, there exist $e \in(N-M) \mathcal{H}, f \in M^{\perp} \mathcal{H}$ and $T, S \in \mathcal{J}$ such that $T e \neq 0 \neq S^{*} f$. If $M \neq N_{-}$, then there is an element $P$ in $\mathcal{N}$ such that $M<P<N$. By the definition of $N$ and $M$, there exist $e \in(P-M) \mathcal{H}$, $f \in(N-P) \mathcal{H}$ and $T, S \in \mathcal{J}$ such that $T e \neq 0 \neq S^{*} f$.

Since $J$ is a J-ideal, $A=T e \otimes f+e \otimes f T \in \mathcal{J}$. Thus $A S=0$. But

$$
A S=T e \otimes f S+e \otimes f T S=T e \otimes f S \neq 0 .
$$

Therefore $N \leq M$, and then

$$
\mathcal{J}=\left(N+N^{\perp}\right) \mathcal{J}\left(N+N^{\perp}\right)=N \mathcal{J} N^{\perp} \subset \mathcal{I}(N) .
$$

By the maximality, we have that $\mathcal{J}=\mathcal{I}(N)$.

Since $\mathcal{I}(N)\left(N \in \mathcal{N}_{0}\right)$ is a maximal nilpotent J-ideal in $\mathcal{T}(\mathcal{N}), \phi(\mathcal{I}(N))$ is also a maximal nilpotent J-ideal in $\mathcal{T}(\mathcal{M})$. By Theorem 7 , there is only one element $\widehat{N} \in \mathcal{M}_{0}$ such that $\phi(\mathcal{I}(N))=\mathcal{I}(\widehat{N})$. Define a map $\widehat{\phi}$ from $\mathcal{N}_{0}$ to $\mathcal{M}_{0}$ by $\widehat{\phi}(N)=\widehat{N}$ for $N \in N_{0}$ such that $\phi\left(\mathcal{I}(N)=\mathcal{I}(\widehat{N})\right.$. Then $\phi\left(N T N^{\perp}\right)=\widehat{N} \phi\left(N T N^{\perp}\right) \widehat{N}^{\perp}$ for every $T \in \mathcal{T}(\mathcal{N})$ and $N \in \mathcal{N}_{0}$.

Proposition 8. The map $\widehat{\phi}$ is bijective.

Proof. First we show that $\hat{\phi}$ is injective. For otherwise, there are $P<Q$ (in $\mathcal{N}_{0}$ ) such that $\phi(\mathcal{I}(P))=\phi(\mathcal{I}(Q))=\mathcal{I}(\widehat{P})$. Choose non-zero vectors $x \in P \mathcal{H}$, $y \in(Q-P) \mathcal{H}$ and $z \in Q^{\perp} \mathcal{H}$. Clearly $\phi(x \otimes y)$ and $\phi(y \otimes z)$ are both in $\mathcal{I}(\widehat{P})$ and hence

$$
\phi(x \otimes y) \phi(y \otimes z)=\phi(y \otimes z) \phi(x \otimes y)=0 .
$$

Applying Proposition 4 to $\phi^{-1}$,

$$
(x \otimes y)(y \otimes z)=0,
$$

but

$$
(x \otimes y)(y \otimes z)=\|y\|^{2} x \otimes z \neq 0 .
$$

Considering $\phi^{-1}$ instead of $\phi$, for every element $M \in \mathcal{M}_{0}, \phi^{-1}(\mathcal{I}(M))$ is a maximal nilpotent J-ideal in $\mathcal{T}(\mathcal{N})$. Hence there is an element $N$ in $\mathcal{N}_{0}$ such that 
$\mathcal{I}(N)=\phi^{-1}(\mathcal{I}(M))$. Thus $\phi(\mathcal{I}(N))=\mathcal{I}(M)$ and hence $M=\widehat{N}$. That is to say, $\widehat{\phi}$ is surjective.

Now we want to identify $\phi(\mathcal{N})$. For that, we need Lemma 9. It seems to be known, but we cannot find a reference.

Lemma 9. Suppose that $S_{1} \in B\left(\mathcal{H}_{1}\right)$ and $S_{2} \in B\left(\mathcal{H}_{2}\right)$ are idempotent operators. If $S_{1} T+T S_{2}=T$ for every $T \in B\left(\mathcal{H}_{2}, \mathcal{H}_{1}\right)$, then either $S_{1}=I$ and $S_{2}=0$ or $S_{1}=0$ and $S_{2}=I$.

Proof. Fix a non-zero vector $y$ in $\mathcal{H}_{2}$. Then for every $x$ in $\mathcal{H}_{1}$, we have

$$
S_{1} x \otimes y+x \otimes y S_{2}=x \otimes y .
$$

This implies that $S_{1}=\lambda I$ for some scalar $\lambda$. Thus the result is immediate from the fact that $S_{1}$ is an idempotent.

Theorem 10. Let $\widehat{N}=\widehat{\phi}(N)$ for $N \in \mathcal{N}_{0}$. Then exactly one of the following holds:

(I) For all $N \in \mathcal{N}, \phi(N)=\left[\begin{array}{ll}I & * \\ 0 & 0\end{array}\right]$ on $\mathcal{H}=\widehat{N} \mathcal{H} \oplus \widehat{N}^{\perp} \mathcal{H}$.

(II) For all $N \in \mathcal{N}, \phi(N)=\left[\begin{array}{ll}0 & * \\ 0 & I\end{array}\right]$ on $\mathcal{H}=\widehat{N} \mathcal{H} \oplus \widehat{N}^{\perp} \mathcal{H}$.

Proof. We first prove that for every $N \in \mathcal{N}_{0}$, one of the following holds:

(a) $\phi(N)=\left[\begin{array}{ll}I & * \\ 0 & 0\end{array}\right]$ on $\mathcal{H}=\widehat{N} \mathcal{H} \oplus \widehat{N}^{\perp} \mathcal{H}$.

(b) $\phi(N)=\left[\begin{array}{ll}0 & * \\ 0 & I\end{array}\right]$ on $\mathcal{H}=\widehat{N} \mathcal{H} \oplus \widehat{N}^{\perp} \mathcal{H}$.

For every $T=N T N^{\perp}$, by Lemma 1(1)

$$
\phi(T)=\phi(N T+T N)=\phi(N) \phi(T)+\phi(T) \phi(N) .
$$

Suppose that $\phi(N)=\left[\begin{array}{cc}S_{1} & * \\ 0 & S_{2}\end{array}\right]$ on $\mathcal{H}=\widehat{N} \mathcal{H} \oplus \widehat{N}^{\perp} \mathcal{H}$. Then $S_{1}$ and $S_{2}$ are idempotent. Since $\phi(T)=\widehat{N} \phi\left(N T N^{\perp}\right) \widehat{N}^{\perp}$, by (7) we have

$$
\phi(T)=S_{1} \phi(T)+\phi(T) S_{2} .
$$

Since $\phi(\mathcal{I}(N))=\mathcal{I}(\widehat{N})$, by Lemma 9 , either $S_{1}=I$ and $S_{2}=0$ which implies (a) holds, or $S_{1}=0$ and $S_{2}=I$ which implies (b) holds.

Suppose that there are $N_{1}$ and $N_{2}$ in $\mathcal{N}_{0}$ such that $\phi\left(N_{1}\right)=\left[\begin{array}{ll}I & * \\ 0 & 0\end{array}\right]$ on $\mathcal{H}=$ $\widehat{N}_{1} \mathcal{H} \oplus \widehat{N}_{1}^{\perp} \mathcal{H}$ and $\phi\left(N_{2}\right)=\left[\begin{array}{l}0 \\ 0 \\ 0\end{array}\right]$ on $\mathcal{H}=\widehat{N}_{2} \mathcal{H} \oplus \widehat{N}_{2}^{\perp} \mathcal{H}$. We consider two cases and reach a contradition.

Case 1. $N_{1}<N_{2}$. Then $N_{1} N_{2}^{\perp}=N_{2}^{\perp} N_{1}=0$ and hence $\phi\left(N_{1}\right) \phi\left(N_{2}^{\perp}\right)=$ $\phi\left(N_{2}^{\perp}\right) \phi\left(N_{1}\right)=0$ by Proposition 4. But $\phi\left(N_{2}^{\perp}\right)=I-\phi\left(N_{2}\right)=\left[\begin{array}{l}I \\ 0 \\ 0\end{array}\right]$ on $\mathcal{H}=$ $\widehat{N}_{2} \mathcal{H} \oplus \widehat{N}_{2}^{\perp} \mathcal{H}$. By a simple computation, if $\widehat{N}_{1} \leq \widehat{N}_{2}$, then $\phi\left(N_{1}\right) \phi\left(N_{2}^{\perp}\right) \neq 0$. If $\widehat{N}_{1}>\widehat{N}_{2}$ (up to now, we don't know whether $\widehat{\phi}$ is order-preserving, i.e. $\widehat{N}_{1}<\widehat{N}_{2}$ ), then $\phi\left(N_{2}^{\perp}\right) \phi\left(N_{1}\right) \neq 0$. This is a contradiction.

Case 2. $N_{1}>N_{2}$. Similarly we can reach a contradiction.

Remark 11. If Theorem 10(I) holds, then $\phi(N) \widehat{N}=\widehat{N}$ and $\widehat{N} \phi(N)=\phi(N)$ for every $N \in \mathcal{N}_{0}$, which implies that $\widehat{N}$ is the projection onto the range of $\phi(N)$. Hence the range of $\phi(N)$ is invariant for $\mathcal{T}(\mathcal{M})$ for every $N \in \mathcal{N}$, therefore for every $T \in \mathcal{T}(\mathcal{N})$ we have that $\phi(T) \phi(N)=\phi(N) \phi(T) \phi(N)$ and

$$
\begin{aligned}
\phi\left(N T N^{\perp}\right) & =\phi\left(N N T N^{\perp} N^{\perp}+N^{\perp} N T N^{\perp} N\right) \\
& =\phi(N) \phi\left(N T N^{\perp}\right) \phi\left(N^{\perp}\right)+\phi\left(N^{\perp}\right) \phi\left(N T N^{\perp}\right) \phi(N) \\
& =\phi(N) \phi\left(N T N^{\perp}\right) \phi\left(N^{\perp}\right) .
\end{aligned}
$$


Hence, since $\phi(N T N)=\phi(N) \phi(T) \phi(N)$ and $\phi\left(N^{\perp} T N^{\perp}\right)=\phi\left(N^{\perp}\right) \phi(T) \phi\left(N^{\perp}\right)$, we have that

$$
\phi\left(N T N^{\perp}\right)=\phi(N) \phi(T) \phi\left(N^{\perp}\right) .
$$

Moreover, in this case $\widehat{\phi}$ is order-preserving. Indeed, let $P<Q$ (in $\mathcal{N}_{0}$ ) and $\widehat{P}=\widehat{\phi}(P)$ and $\widehat{Q}=\widehat{\phi}(Q)$. Choose $x, y, z$ as in Proposition 8. Let $T=x \otimes y$ and $S=y \otimes z$. Then $T S \neq 0$. Since

$$
\phi(S) \phi(T)=\phi(Q) \phi(S) \phi\left(Q^{\perp}\right) \phi(P) \phi(T) \phi\left(P^{\perp}\right)=0,
$$

by Proposition 4,

$$
\phi(T) \phi(S) \neq 0 .
$$

But $\phi(T) \phi(S)=\phi(T) \widehat{P}^{\perp} \widehat{Q} \phi(S)$, so (8) implies that $\widehat{P}^{\perp} \widehat{Q} \neq 0$ and hence $\widehat{P}<\widehat{Q}$.

Similarly, if Theorem 10(II) holds, then $\phi(T) \phi\left(N^{\perp}\right)=\phi\left(N^{\perp}\right) \phi(T) \phi\left(N^{\perp}\right)$ and

$$
\phi\left(N T N^{\perp}\right)=\phi\left(N^{\perp}\right) \phi\left(N T N^{\perp}\right) \phi(N)=\phi\left(N^{\perp}\right) \phi(T) \phi(N) .
$$

Moreover $\widehat{\phi}$ is anti-order-preserving, i.e. if $P<Q$ (in $\mathcal{N}_{0}$ ), then $\widehat{P}>\widehat{Q}$.

In the foregoing, we say that $\phi$ is order preserving if $\widehat{\phi}$ is order preserving and $\phi$ is anti-order preserving if $\widehat{\phi}$ is anti-order preserving.

Lemma 12. We have $\phi\left(\mathcal{N}^{\prime}\right)=\phi(\mathcal{N})^{\prime}$.

Proof. Suppose that $D$ is in $\mathcal{N}^{\prime}$. Then $D N=N D$ for every $N \in \mathcal{N}$.

If $\phi$ is order-preserving, then

$$
\begin{aligned}
& \phi(N) \phi(D)=\phi(N) \phi\left(N D N+N^{\perp} D N^{\perp}\right) \\
& \quad=\phi(N)\left(\phi(N) \phi(D) \phi(N)+\phi\left(N^{\perp}\right) \phi(D) \phi\left(N^{\perp}\right)\right) \\
& \quad=\phi(N) \phi(D) \phi(N)=\phi(D) \phi(N) .
\end{aligned}
$$

So $\phi\left(\mathcal{N}^{\prime}\right) \subset \phi(\mathcal{N})^{\prime}$. On the other hand, suppose that $T$ is in $\phi(\mathcal{N})^{\prime}$. Then $T \phi(N)=$ $\phi(N) T$ for every $N \in \mathcal{N}$ and hence $T \in \mathcal{T}(\mathcal{M})$. Therefore, there is $D \in \mathcal{T}(\mathcal{N})$ such that $T=\phi(D)$. Considering $\phi^{-1}$, we have

$$
\begin{aligned}
& N D=N \phi^{-1}\left(\phi(N) T \phi(N)+\phi\left(N^{\perp}\right) T \phi\left(N^{\perp}\right)\right) \\
& =N\left(N \phi^{-1}(T) N+N^{\perp} \phi^{-1}(T) N^{\perp}\right) \\
& =N D N=D N
\end{aligned}
$$

which implies $D \in \mathcal{N}^{\prime}$ and hence $T \in \phi\left(\mathcal{N}^{\prime}\right)$.

If $\phi$ is anti-order preserving, the proof is similar.

Let $\Omega$ be the subspace spanned by $\mathcal{N}^{\prime}$ and $\left\{\mathcal{I}(N): N \in \mathcal{N}_{0}\right\}$. It is easy to verify that $\Omega$ is in fact an algebra and it contains all rank-1 operators in $\mathcal{T}(\mathcal{N})$. Moreover, using the argument of Lemma 3.11 in [1], we have that:

Lemma 13. Suppose that $\mathcal{N}_{0} \neq \emptyset$. If $\phi$ is order-preserving, then the restriction of $\phi$ to $\Omega$ is multiplicative. If $\phi$ is anti-order preserving, then the restriction of $\phi$ to $\Omega$ is anti-multiplicative.

Lemma 14. Suppose that $\mathcal{N}_{0} \neq \emptyset$. Let $\mathcal{G}$ be a maximal abelian $*$-subalgebra of $\mathcal{T}(\mathcal{N})$. Then $\mathcal{G}$ and $\phi(\mathcal{G})$ are both maximal abelian subalgebras of $B(\mathcal{H})$. 
Proof. Since $\mathcal{G}$ is a $*$-subalgebra of $\mathcal{T}(\mathcal{N})$, it commutes with each $N \in \mathcal{N}$. By the maximality, $\mathcal{N} \subset \mathcal{G}$. Suppose that $T \in B(\mathcal{H})$ such that $T$ commutes with $\mathcal{G}$. Then $T$ commutes with $\mathcal{N}$ and then $T \in \mathcal{T}(\mathcal{N})$. Hence $T \in \mathcal{G}$ and $\mathcal{G}$ is maximal in $B(\mathcal{H})$.

Since $\mathcal{N} \subset \mathcal{G}, \mathcal{G}=\mathcal{G}^{\prime} \subset \mathcal{N}^{\prime}$. By Lemma $13, \phi(\mathcal{G})$ is an abelian subalgebra. Suppose that $X$ belongs to $\phi(\mathcal{G})^{\prime}$; then $X \in \phi(\mathcal{N})^{\prime}$. By Lemma 12, $X=\phi(D)$ for some $D \in \mathcal{N}^{\prime}$ and hence $X \in \phi(\Omega)$. By Lemma 13, the restriction of $\phi^{-1}$ to $\phi(\Omega)$ is multiplicative or anti-muliplicative. Therefore, $D$ commutes with $\mathcal{G}$ and hence $D \in \mathcal{G}$. Thus $X \in \phi(\mathcal{G})$ and $\phi(\mathcal{G})$ is maximal abelian in $B(\mathcal{H})$.

Theorem 15. Suppose that $\phi$ is a Jordan isomorphism from a nest algebra $\mathcal{T}(\mathcal{N})$ onto a nest algebra $\mathcal{T}(\mathcal{M})$. Then there is an invertible operator $S$ such that either $\phi(T)=S T S^{-1}$ or $\phi(T)=S T^{*} S^{-1}$ for every $T \in \mathcal{T}(\mathcal{N})$.

Proof. First we consider the exceptional case where the nest $\mathcal{N}$ is the trivial nest $\{0, I\}$. By Proposition $8, \mathcal{M}$ is also trivial and so $\phi$ is a Jordan automorphism of $B(\mathcal{H})$. Since $B(\mathcal{H})$ is prime ring, it follows from 9 that $\phi$ is either an algebraic automorphism or an anti-automorphism. It is well known that automorphisms of $B(\mathcal{H})$ are spatial. This establishes Therorem 15 in this case.

In the following, we assume that the nest $\mathcal{N}$ is not trivial (i.e. $\mathcal{N}_{0} \neq \emptyset$ ). Let $\Omega$ be as above. By Lemma 13, we only need to consider two cases.

Case 1. The restriction of $\phi$ to $\Omega$ is multiplicative. Let $\mathcal{G}$ be a maximal abelian *-subalgebra of $\mathcal{T}(\mathcal{N})$. Then $\mathcal{G}$ and $\phi(\mathcal{G})$ are maximal abelian in $B(\mathcal{H})$. Hence $\phi(\mathcal{G})$ is norm-closed since the norm closure of $\phi(\mathcal{G})$ is abelian and contains $\phi(\mathcal{G})$. Let $\varphi$ be the restriction of $\phi$ to $\mathcal{G}$. Then $\varphi$ is an isomorphism from the Banach space $\mathcal{G}$ onto $\phi(\mathcal{G})$. Thus for each $D \in \mathcal{G}$,

$$
\sigma(D)=\sigma_{\mathcal{G}}(D)=\sigma_{\varphi(\mathcal{G})}(\varphi(D)),
$$

where $\sigma(D)$ is the spectrum of $D$ in $B(\mathcal{H})$ and $\sigma_{\mathcal{G}}(D)$ is the spectrum of $D$ in $\mathcal{G}$. Since $D$ is normal, $\|\varphi(D)\| \geq\|D\|$. That is, $\varphi^{-1}$ is contractive. Hence, by the Open Mapping Theorem, $\varphi$ is bounded.

Let $\mathcal{U}$ be the set of all unitaries in $\mathcal{G}$. Then $\varphi(\mathcal{U})$ is a bounded abelian group of operators. By a result of Dixmier [4] (also see Corollary 17.2 [3]), there is an invertible operator $T$ such that $T \varphi(\mathcal{G}) T^{-1}$ is a group of unitaries. Since $\mathcal{G}$ is spanned by $\mathcal{U}$, it follows that $T \varphi(\mathcal{G}) T^{-1}$ is spanned by the abelian unitary group $T \varphi(\mathcal{U}) T^{-1}$. Hence $T \varphi(\mathcal{G}) T^{-1}$ is an abelian von Neumann algebra. Clearly, it is maximal abelian in $T \mathcal{T}(\mathcal{M}) T^{-1}$.

For $M \in \mathcal{M}$, let $P_{T M}$ be the orthogonal projection onto the range of $T M$. Let $\mathcal{P}_{\mathcal{M}}=\left\{P_{T M}: M \in \mathcal{M}\right\}$. Then $\mathcal{P}_{\mathcal{M}}$ is a nest of projections on $\mathcal{H}$ and $T \mathcal{T}(\mathcal{M}) T^{-1}=$ $\mathcal{T}\left(\mathcal{P}_{\mathcal{M}}\right)$. By Lemma $14, T \varphi(\mathcal{G}) T^{-1}$ is a maximal abelian $*$-subalgebra. Thus $A d T \circ$ $\varphi$ is an algebraic isomorphim between two maximal abelian $*$-subalgebras, where $A d T \circ \varphi$ means that $A d T \circ \varphi(D)=T \varphi(D) T^{-1}$. Therefore there is a unitary operator $U$ such that $A d T \circ \varphi=A d U$ [5, Chapter III, Part $3 \S 2]$. Let $S_{1}=U^{-1} T$ and $\psi=A d S_{1} \circ \phi$. Then $\psi$ is a Jordan isomorphism from $\mathcal{T}(\mathcal{N})$ onto $S_{1} \mathcal{T}(\mathcal{N}) S_{1}^{-1}$. Moreover the restriction of $\psi$ to $\Omega$ is multiplicative and $\psi(D)=D$ for every $D \in \mathcal{G}$.

Since $S_{1} \mathcal{T}(\mathcal{N}) S_{1}^{-1}$ is a nest algebra, by Remark 11, its corresponding nest is $\psi(\mathcal{N})=\mathcal{N}$ and hence $S_{1} \mathcal{T}(\mathcal{N}) S_{1}^{-1}=\mathcal{T}(\mathcal{N})$. Moreover $\psi\left(\mathcal{N}^{\prime}\right)=\psi(\mathcal{N})^{\prime}=\mathcal{N}^{\prime}$ and $\psi(\mathcal{I}(N))=\mathcal{I}(N)$ for every $N \in \mathcal{N}_{0}$, so $\psi(\Omega)=\Omega$. Hence the restriction of $\psi$ to $\Omega$, still denoted by $\psi$, is an isomorphism onto $\Omega$. Since $\Omega$ contains all rank-1 operators in $\mathcal{T}(\mathcal{N})$, by Theorem 4.1 of [7], there is an invertible operator $S_{2}$ such that $\psi(T)=S_{2} T S_{2}^{-1}$ for every $T \in \Omega$. Let $S=S_{1}^{-1} S_{2}$. Then for every $T \in \Omega$ we 
have that $\phi(T)=S T S^{-1}$. In particular, for every $x \otimes y$ in $\mathcal{T}(\mathcal{N})$, we have that $\phi(x \otimes y)=S x \otimes y S^{-1}$.

Suppose $T \in \mathcal{T}(\mathcal{N})$. If $T$ is a scalar multiple of $I$, then clearly $\phi(T)=S T S^{-1}$. So we assume that $T$ is not a scalar multiple of $I$. Let $\mathcal{N}_{1}=\{N \in \mathcal{N}: N \neq$ 0 and $\left.N_{-}<I\right\}$. Let $N$ be an arbitrary element in $\mathcal{N}_{1}$. Fix a non-zero vector $y$ in $N_{-}^{\perp} \mathcal{H}$. Then $x \otimes y$ is in $\mathcal{T}(\mathcal{N})$ for every $x \in N \mathcal{H}$. Hence we have that

$$
\begin{array}{r}
S T x \otimes y S^{-1}+S x \otimes y T S^{-1}=\phi(T x \otimes y+x \otimes y T) \\
=\phi(T) S x \otimes y S^{-1}+S x \otimes y S^{-1} \phi(T),
\end{array}
$$

and then there is a scalar $\lambda(N)$ such that

$$
\phi(T) S x-S T x=\lambda(N) S x, \quad x \in N \mathcal{H} .
$$

For $N_{1}$ and $N_{2}$ in $\mathcal{N}_{1}$, we have that

$$
\lambda\left(N_{1}\right) S x=\phi(T) S x-S T x=\lambda\left(N_{2}\right) S x, \quad x \in\left(N_{1} \mathcal{H}\right) \cap\left(N_{2} \mathcal{H}\right),
$$

and consequently $\lambda\left(N_{1}\right)=\lambda\left(N_{2}\right)$ since $N_{1}<N_{2}$ or $N_{1} \geq N_{2}$. Thus there is a scalar $\lambda$ such that

$$
\phi(T) S x-S T x=\lambda S x
$$

on $\left\{N \mathcal{H}: N \in \mathcal{N}_{1}\right\}$. But $\bigvee\left\{N \mathcal{H}: N \in \mathcal{N}_{1}\right\}=\mathcal{H}$, so

$$
\phi(T)=S T S^{-1}+\lambda .
$$

Now we show that $\lambda=0$. If $\lambda \neq 0$, for every rank-1 operator $x \otimes y \in \mathcal{T}(\mathcal{N})$, we have

$$
\begin{aligned}
& S T x \otimes y T S^{-1}=\phi(T x \otimes y T)=\phi(T) \phi(x \otimes y) \phi(T) \\
& \quad=\left(\lambda+S T S^{-1}\right) S x \otimes y S^{-1}\left(\lambda+S T S^{-1}\right) \\
& \quad=\lambda^{2} S x \otimes y S^{-1}+\lambda S x \otimes y T S^{-1}+\lambda S T x \otimes y S^{-1}+S T x \otimes y T S^{-1} .
\end{aligned}
$$

Since $\lambda \neq 0$, we have that

$$
T x \otimes y=-x \otimes\left(\bar{\lambda} I+T^{*}\right) y .
$$

By a similar argument as above, there is a scalar $\mu$ such that $S T=\mu S$ and hence $T=\mu I$ which contradicts the assumption. So $\lambda=0$ and then $\phi(T)=S T S^{-1}$.

Case 2. The restriction of $\phi$ to $\Omega$ is anti-multiplicative. Define $\Phi(T)=\phi(T)^{*}$. Then $\Phi$ is a Jordan isomorphism from $\mathcal{T}(\mathcal{N})$ onto $\mathcal{T}\left(\mathcal{M}^{\perp}\right)$. Since the restriction of $\phi$ to $\Omega$ is anti-multiplicative, the restriction of $\Phi$ to $\Omega$ is multiplicative. By Case 1, there is an invertible operator $S$ such that $\Phi(T)=S T S^{-1}$. Thus $\phi(T)=$ $\left(S^{*}\right)^{-1} T^{*} S^{*}$

Remark 16. As a corollary of Theorem 15, we can conclude another result for Jordan isomorphisms of nest algebras: Every Jordan isomorphism between nest algebras is continuous.

\section{ACKNOWLEDGMENT}

The author would like to thank the referee for his very helpful comments and a careful reading of the paper. 
Note. After we submitted this paper we became aware of the recent paper [2] which proved that Jordan isomorphisms of triangular matrix algebras over a connected commutative ring are of the form stated above. So our result was covered by [2] for the special case in which the nest algebras under consideration are upper triangular matrix algebras over the complex numbers. In fact, 2 covers the present paper only in this case since the ring considered in [2] must contain no non-trivial idempotents and must be commutative.

\section{REFERENCES}

1. J. Arazy and B. Solel, Isometries of non-adjoint operators algebras, J. Funct. Anal. 90 (1990), 284-305. MR 91c:47085

2. K.I. Beidar, M. Bresar, M.A. Chebotar, Jordan isomorphisms of triangular matrix algebras over a connected commutative ring, Linear algebra Appl. 312 (2000), 197-201. MR 2001a:16048

3. K.R. Davidson, Nest Algebras, Pitman Research Notes in Mathematics Series 191, Longman Scientific and Technical, Burnt Mill Harlow, Essex, UK, 1988. MR 90f:47062

4. J. Dixmier, Les moyennes invariant dans les semi-groupes et leur applications, Acta Sci. Math. (Szeged) 12A (1950), 213-227.

5. J. Dixmier, Les algèbras d'opèrateurs dans l'espace Hilbertien, Gauthier-Villars, Paris, 1969. MR 50:5482 reprinted MR 98a:46065

6. N. Jacobson and Rickart, Jordan homomorphisms of rings, Trans. Amer. Math. Soc. 69 (1950), 479-502. MR 12:387h

7. J. R. Ringrose, On some algebras of operators II, Proc. London Math. Soc. 16 (1966), 385-402. MR 33:4703

8. P. Semrl, Jordan *-derivations of standard operator algebras, Proc. Amer. Math. Soc. 120 (1994), 515-518. MR 94d:46066

9. M.F. Smiley, Jordan homomorphisms onto prime rings, Trans. Amer. Math. Soc. 84 (1957), 426-429. MR 18:715b

10. B. Solel, Isometries of CSL algebras, Trans. Amer. Math. Soc. 332 (1992), 595-606. MR 92j:47082

Department of Mathematics, Suzhou University, Suzhou 215006, People's Republic OF CHINA

E-mail address: fylu@pub.sz.jsinfo.net 